\title{
PAUL SABLONNIERE
}

\section{Une méthode de résolution numérique de certaines équations intégrales de type Hammerstein}

Revue française d'automatique, informatique, recherche opérationnelle. Analyse numérique, tome 9, no 1 (1975), p. 105-118.

<http://www.numdam.org/item?id=M2AN_1975_9_1_105_0>

(C) AFCET, 1975, tous droits réservés.

L'accès aux archives de la revue "Revue française d'automatique, informatique, recherche opérationnelle. Analyse numérique » implique l'accord avec les conditions générales d'utilisation (http://www.numdam.org/legal. php). Toute utilisation commerciale ou impression systématique est constitutive d'une infraction pénale. Toute copie ou impression de ce fichier doit contenir la présente mention de copyright.

\section{Numdam}




\title{
UNE METHODE DE RESOLUTION NUMERIQUE DE CERTAINES EQUATIONS INTEGRALES DE TYPE HAMMERSTEIN
}

\author{
par Paul SABLONNIERE * \\ Communiqué par P.J. LAURENT
}

Résumé. - On utilise la technique du plongement invariant pour résoudre numériquement certaines équations intégrales de type Hammerstein, le paramètre étant la borne supérieure de l'intégrale. On en déduit un système d'équations différentielles que l'on intègre par des méthodes numériques classiques.

\section{I - INTRODUCTION}

1.1. - Soient $[a, b]$ un intervalle de $\mathbb{R}, C[a, b]$ l'espace de BANACH des fonctions réelles continues muni de la norme du maximum. On cherche à résoudre l'équation intégrale :

$$
y(t)=f(t)+\lambda \int_{a}^{b} K(t, s) \phi(s, y(s)) d s
$$

avec les hypothèses suivantes :

H1) $f$ est une fonction réelle continue sur $[a, b]$ et $K$ une fonction réelle continue sur $[a, b] \times[a, b]$.

H2) Pour tout $f \in C[a, b]$ et tout $r>0$, on pose :

$$
\begin{aligned}
& D(f, r)=\left\{(s, u) \in \mathbb{R}^{2} \mid a \leqslant s \leqslant b \text { et } f(s)-r \leqslant u \leqslant f(s)+r\right\} \\
& B(f, r)=\{y \in C[a, b] \mid\|y-f\| \leqslant r\}
\end{aligned}
$$

On suppose que $\phi(s, u)$ est une fonction réelle continue sur $D(f, r)$ et possèce une dérivée partielle $\frac{\partial \phi}{\partial u}(s, u)$ (notée $\phi$ ' dans la suite), lipschitzienne par

* I.U.T. Informatique - Lille

Revue Française d'Automatique, Informatique et Recherche Opérationnelle $n^{\circ}$ avril 1975, R-1. 
rapport à $u$; plus précisément, il existe $L_{\phi^{\prime}}>0$ tel que, pour tous $\left(s, u_{1}\right)$ et $\left(s, u_{2}\right)$ de $D(f, r)$ :

$$
\left|\phi^{\prime}\left(s, u_{1}\right)-\phi^{\prime}\left(s, u_{2}\right)\right| \leqslant \mathrm{L}_{\phi},\left|u_{1}-u_{2}\right|
$$

H3) On choisit $\lambda \in \mathbb{R}$ tel que :

$$
|\lambda|<\frac{1}{M_{K}(b-a)} \min \left(\frac{r}{M_{\phi}}, \frac{1}{M_{\phi}}\right)
$$

où $M_{K}, M_{\phi}$ et $M_{\phi}$, désignent les maxima des fonctions $|K(t, s)|,|\phi(s, u)|$ et $\left|\phi^{\prime}(s, u)\right|$ sur leurs ensembles de continuité respectifs.

Ces hypothèses permettent de prouver le résultat suivant :

\begin{tabular}{l|l} 
Théorème 1. - & $\begin{array}{l}\text { Si } r>0 \text { est donné, l'équation (1) admet une solution unique dans } \\
B(f, r) .\end{array}$
\end{tabular} Démonstration. -

Désignons par $P$ l'opérateur qui à $y \in C[a, b]$ fait correspondre $P y \in C[a, b]$ défini par :

$$
P y(t)=f(t)+\lambda \int_{a}^{b} K(t, s) \phi(s, y(s)) d s
$$

Si $y \in B(f, r)$, alors $(s, y(s)) \in D(f, r)$ pour tout $s \in[a, b]$, donc $\phi(s, y(s))$ a un sens et on a :

$$
|| P y-f|| \leqslant|\lambda|(b-a) M_{K} M_{\phi}<r \text { d'après (H3) }
$$

On constate que $P$ applique la boule $B(f, r)$ dans elle-même (et même dans son intérieur).

D'autre part, pour $y_{1}$ et $y_{2} \in B(f, r)$ :

$$
|| P y_{1}-P y_{2}|| \leqslant|\lambda|(b-a) M_{K} M_{\phi},|| y_{1}-y_{2}||
$$

et comme (H3) entraîne : $|\lambda|(b-a) M_{K} M_{\phi},<1$, l'opérateur $P$ est contractant, donc il admet un point fixe dans la boule $B(f, r)$, c.q.f.d. (Cf. [1], [2], [6]).

1.2. -Associons maintenant à l'équation (1) la famille d'équations dépendant du paramètre $\mathrm{x} \in[a, b]$ :

Revue Française d'Automatique, Informatique et Recherche Opérationnelle 
(2)

$$
y_{x}(t)=f(t)+\lambda \int_{a}^{x} K(t, s) \phi\left(s, y_{x}(s)\right) d s
$$

Pour chaque valeur de $x$, cette équation a une solution unique $y_{x}$ dans la boule $B(f, r)$, l'hypothèse (H3) entraînant la contraction de chaque opérateur intégral en $x$.

Nous allons montrer que la fonction $y_{*}: x \rightarrow y_{x}$ définie sur $[a, b]$ et à valeurs dans $B(f, r)$ est dérivable et vérifie une équation différentielle de type initial que l'on pourra résoudre numériquement par des méthodes par pas.

Auparavant, nous étudions une famille d'équations intégrales linéaires liées à l'équation (1).

\section{II - ETUDE D'UNE FAMILLE D'EQUATIONS LINEAIRES}

Posons, pour toute fonction $\psi \in B(f, r)(t, s, z \in[a, b])$ :

$$
\begin{aligned}
& H(t, s ; \psi)=K(t, s) \phi^{\prime}(s, \psi(s)) \\
& F(t, z ; \psi)=K(t, z) \phi(z, \psi(z))
\end{aligned}
$$

L'équation intégrale linéaire suivante, où $z$ est fixé dans $[a, b]$

$$
\Theta_{z}(t ; \psi)=\lambda F(t, z ; \psi)+\lambda \int_{a}^{z} H(t, s ; \psi) \Theta_{z}(s ; \psi) d s,
$$

a un noyau vérifiant, en vertu de (H3) :

$$
|\lambda|(z-a) \max _{(t, s) \in[a, b]^{2}}|H(t, s ; \psi)|<1
$$

Par conséquent, elle admet un noyau résolvant $\Gamma_{z}(\mathrm{t}, \mathrm{s} ; \psi)$ et une solution unique $\tau_{z}(t ; \psi)$ vérifiant le système différentiel de type initial (Cf [3]).

$$
\begin{aligned}
& \frac{\partial}{\partial z} \tau_{z}(t ; \psi)=\lambda \Gamma_{z}(t, z ; \psi) \tau_{z}(z ; \psi) \\
& \tau_{a}(t ; \psi)=\lambda F(t, a ; \psi) \\
& \frac{\partial}{\partial z} \Gamma_{z}(t, s ; \psi)=\lambda \Gamma_{z}(t, z ; \psi) \Gamma_{z}(z, s ; \psi) \\
& \Gamma_{a}(t, s ; \psi)=H(t, s ; \psi)
\end{aligned}
$$

$\mathrm{n}^{\circ}$ avril 1975, R-1. 
La solution de (5) est donnée également par :

$$
\tau_{z}(t ; \psi)=\lambda F(t, z ; \psi)+\lambda \int_{a}^{z} \Gamma_{z}(t, u ; \psi) F(u, z ; \psi) d u
$$

ou encore

$$
\tau_{z}(t ; \psi)=\lambda \phi(z, \psi(z))\left[K(t, z)+\lambda \int_{a}^{z} \Gamma_{z}(t, u ; \psi) K(u, z) d u\right]
$$

Nous sommes amenés à introduire la fonction :

$$
\gamma_{z}(t, s ; \psi)=K(t, s)+\lambda \int_{a}^{z} \Gamma_{z}(t, u ; \psi) K(u, s) d u
$$

Il est facile de voir que :

$$
\Gamma_{z}(t, s ; \psi)=\phi^{\prime}(s, \psi(s)) \cdot \gamma_{z}(t, s ; \psi)
$$

et que la fonction $\gamma_{z}(t, s ; \psi)$ est solution de :

$$
\begin{aligned}
& \frac{\partial}{\partial z} \gamma_{z}(t, s ; \psi)=\phi^{\prime}(z, \psi(z)) \gamma_{z}(t, z ; \psi) \gamma_{z}(z, s ; \psi) \\
& \gamma_{a}(t, s ; \psi)=K(t, s)
\end{aligned}
$$

En vertu de l'égalité (7), la solution de (5) ou de (6) est donnée sans calculs par :

$$
\tau_{z}(t ; \psi)=\lambda \phi(z, \psi(z)) \gamma_{z}(t, z ; \psi)
$$

Dès que l'on connaît $\gamma_{z}$ solution de (10).

Nous allons étudier quelques propriétés de $\gamma_{z}$ et de $\tau_{z}$.

\section{1. - Existence et unicité de la solution de (10)}

Il existe au moins une solution puisque la relation (8) exprime $\gamma_{z}$ en fonction de $\Gamma_{z}$, résolvant de l'équation intégrale (5).

Pour l'unicité, nous écrivons (10) sous la forme :

$$
\gamma_{z}(t, s ; \psi)=K(t, s)+\lambda \int_{a}^{z} \phi^{\prime}(u, \psi(u)) \cdot \gamma_{u}(t, u ; \psi) \gamma_{u}(u, s ; \psi) d u
$$

Si cette équation admet deux solutions bornées ${ }_{\gamma}^{1}$ et ${ }_{\gamma}^{2}$ avec $\left|{ }_{\gamma}^{1}\right|$ et $|\underset{\gamma}{2}| \leqslant M_{\gamma}(\psi)$ 
pour $(z, s, t) \in[a, b]^{3}$ et $\psi \in B(f, r)$ fixée, on peut écrire :

$\max _{t, s}\left|\frac{1}{\gamma_{z}}(t, s ; \psi)-\gamma_{z}(t, s ; \psi)\right| \leqslant 2|\lambda| M_{\phi}, M_{\gamma}(\psi) \int_{a}^{z} \max _{t, s}\left|\gamma_{u}(t, s ; \psi)-\gamma_{u}^{2}(t, s ; \psi)\right| d u$

Un lemme classique de majoration nous donne immédiatement :

$$
\underset{\gamma_{z}}{1}(t, s ; \psi)=\stackrel{2}{\gamma_{z}}(t, s ; \psi)
$$

Nous pouvons énoncer le :

Théorème 2. - L'équation différentielle :

$$
\begin{aligned}
& \frac{\partial}{\partial z} \gamma_{z}(t, s ; \psi)=\lambda \phi^{\prime}(z, \psi(z)) \cdot \gamma_{z}(t, s ; \psi) \gamma_{z}(z, s ; \psi) \\
& \gamma_{a}(t, s ; \psi)=K(t, s)
\end{aligned}
$$

admet, pour chaque fonction $\psi \in B(f, r)$, une solution unique bornée sur $[a, b]^{3}$.

\section{2. - Evaluation d'une borne uniforme $M_{\gamma}$}

Compte tenu de (8) et des équations intégrales vérifiées par le résolvant $\Gamma_{z}$, on établit que :

$$
\gamma_{z}(t, s ; \psi)=K(t, s)+\lambda \int_{a}^{z} \gamma_{z}(t, u ; \psi) \phi^{\prime}(u, \psi(u)) \cdot K(u, s) d u
$$

qui donne la majoration suivante :

$$
M_{\gamma}(\psi) \leqslant M_{K}+|\lambda|(b-a) M_{\phi}, M_{K} M_{\gamma}(\psi) .
$$

Compte tenu de l'hypothèse $(\mathrm{H} 3)$, on obtient :

$$
\forall \psi \in B(f, r): M_{\gamma}(\psi) \leqslant M_{\gamma}=\frac{M_{K}}{1-|\lambda|(b-a) M_{K} M_{\phi}}
$$

2.3. - Etude de l'application : $\psi \rightarrow \gamma_{z}(t, s ; \psi)$

Soient $\psi_{1}$ et $\psi_{2} \in B(f, r)$. La relation (13) ci-dessus nous permet d'écrire :

$$
\begin{aligned}
\left|\gamma_{z}\left(t, s ; \psi_{1}\right)-\gamma_{z}\left(t, s ; \psi_{2}\right)\right| & \leqslant|\lambda| \int_{a}^{z} \mid \gamma_{z}\left(t, u ; \psi_{1}\right) \phi^{\prime}\left(u, \psi_{1}(u)\right) K(u, s) \\
& -\gamma_{z}\left(t, u ; \psi_{2}\right) \phi^{\prime}\left(u, \psi_{2}(u)\right) K(u, s) \mid d u
\end{aligned}
$$

$\mathrm{n}^{\circ}$ avril 1975, R-1. 
Le second membre est inférieur ou égal à :

$$
\begin{aligned}
|\lambda| M_{\gamma} L_{\phi^{\prime}} \max _{a \leqslant u \leqslant z}\left|\psi_{1}(u)-\psi_{2}(u)\right|(z-a) \cdot M_{K} \\
\quad+|\lambda| M_{\phi}, M_{K} \int_{a}^{z}\left|\gamma_{z}\left(t, u ; \psi_{1}\right)-\gamma_{z}\left(t, u ; \psi_{2}\right)\right| d u
\end{aligned}
$$

On en déduit donc :

$$
\max \left|\gamma_{z}\left(t, s ; \psi_{1}\right)-\gamma_{z}\left(t, s ; \psi_{2}\right)\right| \leqslant L_{\gamma_{a}} \max _{a \leqslant z}\left|\psi_{1}(u)-\psi_{2}(u)\right|
$$

avec

$$
L_{\gamma}=\frac{|\lambda|(b-a) M_{K} M_{\gamma} L_{\phi},}{1-|\lambda|(b-a) M_{K} M_{\phi^{\prime}}}=|\lambda|(b-a) L_{\phi}, M_{\gamma}^{2}
$$

\section{4. - Etude de la solution de l'équation intégrale (5)}

Rappelons (11) que :

$$
\tau_{z}(t ; \psi)=\lambda \phi(z, \psi(z)) \gamma_{z}(t, z ; \psi) .
$$

Les calculs précédents nous conduisent à poser, pour toute fonction $B(f, r)$ et tout $z \in[a, b]$ :

$$
F(z, \psi)=\lambda \phi(z, \psi(z)) \cdot \gamma_{z}(., z ; \psi)=\tau_{z}(. ; \psi)
$$

$F$ est une application de $[a, b] \times B(f, r)$ dans $C[a, b]$.

On a alors le :

Théorème 3. - L'application $F(z, \psi)$ est uniformément lipschitzienne par rapport à $\psi \epsilon B(f, r)$.

Pour tout $z \in[a, b]$ et tout couple $\left(\psi_{1}, \psi_{2}\right)$ de $B(f, r)$, on a :

$$
\left\|F\left(z, \psi_{1}\right)-F\left(z, \psi_{2}\right)\right\| \leqslant L_{F} \max _{a \leqslant u \leqslant z}\left|\psi_{1}(u)-\psi_{2}(u)\right|
$$

$\operatorname{avec} L_{F}=|\lambda|\left(M_{\gamma} \mathrm{M}_{\phi},+M_{\phi} L_{\gamma}\right)$

Démonstration. -

A partir de (11), (H2) et (15), on peut écrire :

$$
\left|\lambda \phi\left(z, \psi_{1}(z)\right) \gamma_{z}\left(t, z ; \psi_{1}\right)-\lambda \phi\left(z, \psi_{2}(z)\right) \gamma_{z}\left(t, z ; \psi_{2}\right)\right|
$$

Revue Française d'Automatique, Informatique et Recherche Opérationnelle 


$$
\begin{aligned}
& \leqslant|\lambda| M_{\gamma} M_{\phi^{\prime}} \max _{a \leqslant u \leqslant z}\left|\psi_{1}(u)-\psi_{2}(u)\right| \\
& +|\lambda| M_{\phi} L_{\gamma_{a \leqslant u \leqslant z}} \max _{1}\left|\psi_{1}(u)-\psi_{2}(u)\right|
\end{aligned}
$$

On peut remarquer également que, pour toute fonction $\psi \in B(f, r)$ et tout $z \in[a, b]$, la solution de l'équation intégrale (5) vérifie :

$$
\max _{a \leqslant t \leqslant b}\left|\tau_{z}(t ; \psi)\right| \leqslant|\lambda| M_{\phi} M_{\gamma}=L_{*}
$$

\section{III - CONTINUITE ET DERIVABILITE DE L'APPLICATION $y_{*}$}

Rappelons que, pour tout $x \in[a, b], y_{*}(x)=y_{x}$ est l'unique solution de l'équation (2) dans la boule $B(f, r)$.

\section{1. - Continuité de $y_{*}$}

Théorème 4. - Les hypothèses $(\mathrm{H} 1),(\mathrm{H} 2)$ et $(\mathrm{H} 3)$ entraînent que $y_{*}$ est continue sur $[a, b]$.

Démonstration. -

$$
\left\|y_{*}(x+h)-y_{*}(x)\right\|=\max _{a \leqslant t \leqslant b}\left|y_{x+h}(t)-y_{x}(t)\right|
$$

Or, cette dernière quantité est majorée par :

$$
|\lambda| M_{K} M_{\phi}|h|+|\lambda| M_{K} M_{\phi}, \int_{a}^{x}\left|y_{x+h}(s)-y_{x}(s)\right| d s
$$

Puisque

$$
|\lambda| M_{K} M_{\phi^{\prime}}(x-a) \leqslant|\lambda| M_{K} M_{\phi^{\prime}}(b-a)<1
$$

On en déduit :

$$
\left\|y_{*}(x+h)-y_{*}(x)\right\| \leqslant \frac{|\lambda| M_{K} M_{\phi}}{1-|\lambda| M_{K} M_{\phi^{\prime}}(b-a)}|h|
$$

ou encore, compte tenu de (18):

$$
\left\|y_{*}(x+h)-y_{*}(x)\right\| \leqslant L_{*}|h|
$$

$\mathrm{n}^{\circ}$ avril $1975, \mathrm{R}-1$. 


\section{2. - Dérivabilité de $y_{*}$}

Nous allons construire une application $\mathrm{y}^{\prime} \mathrm{de}[a, b]$ dans $\mathrm{C}[a, b]$ qui vérifie :

$$
\mathrm{V} \epsilon>0 \mathrm{~g} \alpha(\epsilon)>0 \text { tel que }|\mathrm{h}|<\alpha(\epsilon)
$$

entraîne :

$$
|| y_{*}(x+h)-y_{*}(x)-h y_{*}^{\prime}(x)|| \leqslant \epsilon|h|
$$

Puisque $y_{*}(x)=y_{x} \in B(f, r)$, l'équation intégrale linéaire (5) du paragraphe précédent a une solution unique $\tau_{x}\left(t ; y_{x}\right)$ quand on prend $\psi=y_{x}$ et $z=x$. Enonçons le :

Théorème 5. - L'application $y_{*}$ a pour dérivée sur $[a, b]$ la fonction $y_{*}^{\prime}$ telle que $y_{*}^{\prime}(x)$ soit solution de l'équation intégrale linéaire (5) où $z=x$ et $\psi=y_{x}$.

\section{Démonstration. -}

Si on pose $y_{*}^{\prime}(x)=\tau_{x}\left(t ; y_{x}\right)^{\prime}(19)$ s'écrit :

$$
\max _{a \leqslant t \leqslant b}\left|y_{x+h}(t)-y_{x}(t)-h \tau_{x}\left(t ; y_{x}\right)\right| \leqslant \epsilon|h|
$$

Or, le premier membre est majoré par :

$$
\begin{gathered}
|\lambda|\left|\int_{x}^{x+h} K(t, s) \phi\left(s, y_{x+h}(s)\right) d s-h K(t, x) \phi\left(x ; y_{x}(x)\right)\right| \\
+|\lambda| M_{K} \int^{x}\left|\phi\left(s, y_{x+h}(s)\right)-\phi\left(x, y_{x}(s)\right)-\mathrm{h} \phi '\left(s, y_{x}(s)\right) \tau_{x}\left(s ; y_{x}\right)\right| d s
\end{gathered}
$$

La première valeur absolue est majorée par une quantité de la forme $|h| \omega_{1}(h)$ où $\omega_{1}(h) \rightarrow 0$ avec $h$ uniformément par rapport à $x$ : ceci résulte de la continuité uniforme de $K, \phi$ et $y_{*}$ sur leurs domaines respectifs.

La deuxième valeur absolue est majorée par :

$$
\begin{gathered}
|\lambda| M_{K} \int_{a}^{x} \mid\left(\Delta_{h} y_{x}(s) \phi^{\prime}\left(s, y_{x}(s)+\Delta_{h} y_{x}(s) \cdot \Theta(s)\right)-h y^{\prime}\left(s, y_{x}(s)\right) \tau_{x}\left(s ; y_{x}\right) \mid d s\right. \\
\text { où } \Delta_{h} y_{x}(s)=y_{x+h}(s)-y_{x}(s) \text { et } 0<\Theta(s)<1 .
\end{gathered}
$$


Cette quantité peut être majorée à son tour par :

$$
\begin{gathered}
|\lambda| M_{K} M_{\phi}, \int_{a}^{x}\left|y_{x+h}(s)-y_{x}(s)-h \tau_{x}\left(s ; y_{x}\right)\right| d s \\
+|\lambda| M_{K}(x-a)|h| L \max _{a \leqslant s \leqslant x}\left|\phi^{\prime}\left(s, y_{x}(s)+\Theta(s) \Delta_{h} y_{x}(s)\right)-\phi^{\prime}\left(s, y_{x}(s)\right)\right|
\end{gathered}
$$

Comme $\left|\Delta_{h} y_{x}(s)\right| \leqslant L_{*}|h|$, on a aussi :

$$
\left|\phi^{\prime}\left(s, y_{x}(s)+\Theta(s) \Delta_{h} y_{x}(s)\right)-\phi^{\prime}\left(s, y_{x}(s)\right)\right| \leqslant L_{\phi},\left|\Delta_{h} y_{x}(s)\right| \leqslant L_{\phi}, L_{*}|h|
$$

En résumé, on obtient :

$$
\begin{aligned}
& \left|y_{x+h}(t)-\mathrm{y}_{x}(t)-h \tau_{x}\left(t ; y_{x}\right)\right| \leqslant|h|\left[\omega_{1}(h)+\omega_{2}(h)\right] \\
& +|\lambda| M_{K} M_{\phi}, \int_{a}^{x}\left|y_{x+h}(s)-y_{x}(s)-h \tau_{x}\left(s ; y_{x}\right)\right| d s .
\end{aligned}
$$

où l'on a posé $\omega_{2}(h)=|\lambda| M_{K}(b-a) L_{\phi}, L_{*}^{2}|h|$

Cette majoration conduit à :

$$
\left\|y_{*}(x+h)-y_{*}(x)-h y_{*}^{\prime}(x)\right\| \leqslant \frac{\omega_{1}(h)+\omega_{2}(h)}{1-|\lambda| M_{K} M_{\phi^{\prime}}(b-a)}|h|
$$

Comme $\omega_{1}$ et $\omega_{2}$ sont indépendants de $x$, on voit que :

$$
\mathrm{V} \epsilon>0 \exists \alpha(\epsilon) \text { t.q. }|h|<\alpha(\epsilon) \Rightarrow \frac{\left(\omega_{1}+\omega_{2}\right)(h)}{1-|\lambda| M_{K} M_{\phi},(b-a)}<\epsilon
$$

Ceci prouve bien que $y_{*}^{\prime}(x)=\tau_{x}\left(. ; y_{x}\right)$, c.q.f.d.

\section{3. - Equation différentielle vérifiée par $y_{*}$.}

Compte tenu des théorèmes 2,3 et 5 , nous pouvons énoncer le résultat suivant :

Théorème 6. - Soit l'équation fonctionnelle :

$$
\text { (20) }\left\{\begin{array}{l}
y_{*}^{\prime}(x)=F\left(x ; y_{*}(x)\right) \\
y_{*}(a)=f
\end{array}\right.
$$

$\mathrm{n}^{\circ}$ avril $1975, \mathrm{R}-1$. 
avec $F(x, \psi)=\lambda \phi(x, \psi(x)) \gamma_{x}(., x ; \psi)$ où $\gamma_{x}$ est la solution unique de :

$$
\begin{array}{ll}
\frac{\partial}{\partial z} \gamma_{z}(t, s ; \psi)=\lambda \phi^{\prime}(z, \psi(z)) & \gamma_{z}(t, z ; \psi) \gamma_{z}(z, s ; \psi) \\
\gamma_{a}(t, s ; \psi)=K(t, s) & \text { pour } a \leqslant z \leqslant x
\end{array}
$$

Alors (20) admet une solution unique sur $[a, b]$ et, pour tout $x \in[a, b]$, $y_{*}(x)=y_{x}$ est la solution unique de l'équation (2). En particulier $y_{*}(b)$ est la solution de (1).

Il y a donc équivalence entre le problème intégral (2) et le problème (20) ci-dessus, moyennant le calcul de $\gamma_{x}\left(t, x, y_{x}\right)$ pour chaque valeur de $x \in[a, b]$.

\section{IV - RESOLUTION NUMERIQUE}

\section{1. - Equation (10)}

Cette équation généralise l'équation résolue dans [3], [7] et [9] au moyen de méthodes à pas séparés de type RUNGE-KUTTA. Lorsque $\phi(s, u)=u, \phi^{\prime}(s, u)=1$, et on retrouve l'équation de la résolvante des équations linéaires de 2 ème espèce.

Pour le formalisme, nous renvoyons aux références ci-dessus.

\section{2. - Equation (20)}

Contrairement à ce qui se passe pour un problème initial classique du type :

$$
\left\{\begin{array}{l}
y^{\prime}=f(x, y) \\
y(a)=y_{0}
\end{array}\right.
$$

on ne connaît qu'une expression approchée de $F\left(x, y_{*}\right)$ qui est calculée au moyen de l'équation (10). En généralisant un résultat de HENRICI [8], sur les équations du type ci-dessus, on montre le résultat suivant :

Supposons que, pour toute fonction $\psi \in B(f, r)$, tout $h>0$ assez petit, on ait obtenu :

$$
F(x, \psi)=F(x, \psi)+h^{q} \epsilon(h)
$$


au moyen d'une méthode à pas séparés de rang $q$ (où $\epsilon(h)$ reste bornée quand $h \rightarrow 0$ ).

Dans ce cas si on calcule $y_{*}$ au moyen d'une méthode à pas séparés de rang $p$, l'erreur sera de l'ordre de $h^{r}$ où $r=\min (p, q)$.

Pratiquement, on a donc intérêt à utiliser des méthodes de même rang pour résoudre (10) et (20).

\section{$V$ - EXEMPLE}

\section{1. - Résultats théoriques}

Proposons-nous de chercher des conditions suffisantes sur $\alpha$ et $\lambda \in \mathbb{R}$ pour que l'équation :

$$
y(t)=\alpha t+\lambda \int_{0}^{1} t s[y(s)]^{2} d s
$$

ait une solution réelle unique dans la boule $B(f, r)$ où $f(t)=\alpha t$ et $r>0$ est donné.

Les solutions de (21) sont de la forme $y(t)=k t$, ce qui conduit à l'équation :

$$
\lambda k^{2}-4 k+4 \alpha=0
$$

Comme $\Delta^{\prime}=4-4 \alpha \lambda$, on voit que $k$ sera réel si l'on choisit $\alpha$ et $\lambda \epsilon \mathbb{R}$ tels que :

$$
\alpha \lambda \leqslant 1
$$

Calculons maintenant la valeur maximale de

$$
\lambda_{0}(r)=\frac{1}{M_{K}(b-a)} \min \left(\frac{r}{M_{\phi}}, \frac{1}{M_{\phi}}\right)
$$

intervenant dans l'hypothèse (H3), sachant que nous avons ici $M_{K}=1$ et $b-a=1$ et qu'un calcul simple donne :

$$
\frac{r}{M_{\phi}}=\frac{r}{(|\alpha|+r)^{2}} \text { et } \frac{1}{M_{\phi}}=\frac{1}{2(|\alpha|+r)}
$$

La valeur maximale de ces deux quantités étant atteinte pour $r=|\alpha|$, on en déduit que :

$$
\max _{r>0} \lambda_{0}(r)=\frac{1}{4|\alpha|}
$$

$\mathrm{n}^{\circ}$ avril $1975, \mathrm{R}-1$. 
La condition suffisante $(\mathrm{H} 3)$ est donc vérifiée si :

$$
|\alpha \lambda|<\frac{1}{4}
$$

qui est nettement plus restrictif que (23).

\section{2. - Cas particulier}

Si nous prenons $\alpha$ et $\lambda>0$, nous aurons une seule solution dans la boule $B(f, \alpha)$ de $C[0,1]$ si $\lambda \alpha<\frac{1}{4}$; cette solution est :

$$
y(t)=\frac{2}{\lambda}(1-\sqrt{1-\lambda \alpha}) t
$$

Prenons, par exemple $\alpha=1$ et $\lambda=\frac{1}{8}$ :

$$
y(t)=t+\frac{1}{8} \int_{0}^{1} t . s .[y(s)]^{2} d s
$$

La solution unique dans la boule $B(t, 1)$ est :

$$
y(t)=(16-4 \sqrt{14}) t \simeq 1,03337 t
$$

\section{3. - Variations de la solution en fonction de $x \in[0,1]$}

Considérons l'équation

$$
y_{x}(t)=t+\frac{1}{8} \int_{0}^{x} \text { t.s. }\left[y_{x}(s)\right]^{2} d s
$$

La solution dans la boule $B(t, 1)$ est de la forme :

$$
y_{x}(t)=k(x) t
$$

où $k(x)$ est solution de :

$$
x^{4} k^{2}(x)-32 k(x)+32=0
$$

Comme $\Delta^{\prime}=256-32 x^{4}$, il y aura au moins une solution réelle si $x^{4} \leqslant 8$, soit $x \leqslant 1,68$ environ.

Cette solution est nécessairement :

$$
k(x)=\frac{16}{x^{4}}\left(1-\sqrt{1-\frac{x^{4}}{8}}\right)
$$


On constate que $k^{*}(x)=\frac{16}{x^{4}}\left(1+\sqrt{1-\frac{x^{4}}{8}}\right)$ ne convient pas car $k^{*}(x) \rightarrow+\infty$ quand $x \rightarrow 0$ tandis que $k(x) \stackrel{\rightarrow}{\rightarrow}$.

\section{4. - Solution numérique.}

L'essai a été fait avec un pas $k=\frac{1}{8}$ en utilisant la méthode d'Euler modifiée (méthode de Runge-Kutta d'ordre 2).

\begin{tabular}{|l|l|l|l|l|l|l|l|l|}
\hline \multicolumn{1}{|c|}{$t$} & 0,125 & 0,25 & 0,375 & 0,5 & 0,625 & 0,750 & 0,875 & 1 \\
\hline 0,25 & 0,4 & 0,07 & 0,11 & 0,15 & 0,19 & 0,23 & 0,27 & 0,30 \\
0,5 & 0,15 & 0,37 & 0,46 & 0,61 & 0,76 & 0,92 & 1,07 & 1,22 \\
0,75 & 0,34 & 0,69 & 1,03 & 1,38 & 1,72 & 2,07 & 2,41 & 2,76 \\
1 & 0,56 & 1,13 & 1,69 & 2,26 & 2,83 & 3,39 & 3,96 & 4,53 \\
\hline
\end{tabular}

(On donne dans ce tableau, pour différentes valeurs de $x$, les erreurs absolues multipliées par $10^{4}$ )

Dans [5] et [11], on a donné des résultats numériques pour l'équation :

$$
y(t)=\frac{3}{4} t+\int_{0}^{1} t s[y(s)]^{2} d s
$$

qui ne vérifie pas l'hypothèse $(\mathrm{H} 3)$ sur $\lambda$. Les résultats numériques obtenus sont les suivants (on donne l'erreur en valeur absolue multipliée par $10^{4}$ pour $x=1$ ).

\begin{tabular}{|c|c|c|c|c|c|c|c|c|c|}
\hline$t$ & 0 & 0,125 & 0,25 & 0,375 & 0,5 & 0,625 & 0,75 & 0,875 & 1 \\
\hline $10^{4} x$ err. & 0 & 10,5 & 21 & 31,5 & 42 & 52,6 & 63,1 & 73,6 & 84,2 \\
\hline
\end{tabular}




\section{BIBLIOGRAPHIE}

[ 1] L. V. KANTOROVitch - G P. AKilov. Functional analysts in normed spaces. Pergamon press, (1964)

[ 2] M A KRASNOSElSkil, Topological methods in the theory of monlinear integral equations. Pergamon press (1964)

[ 3] P. POUZET - C. BRoudiscou, Traltement numerique des equations integrales lınéaires de Fredholm à résolvante partielle continue $\mathrm{C} \mathrm{R}$ Acad. Sc Paris, Sérıe A-B 268 (1968) A 1279-A 1281.

[ 4] P MOREL. Résolution de $f(x)=0$ par intégration d'une équation différentıelle sur un intervalle finı. Séminarre d'Analyse Numérique, Lyon (19691970).

[ 5] P SABLONNIERE. Système differentıel assocle à certaines équatıons intégrales de type Hammerstein. C.R. Acad. Sc. Parıs, Séne A-B 277 (1973) A 605 1607.

[ 6] P.M. ANSELONE. Non linear integral equations. The University of Wisconsin Press, Madison (1964).

[ 7] P POUZET Methodes numériques par pas pour le traltement de certaines équatıons intégrales lınéaires de Fredholm, Colloque d'Analyse Numérıque, Aussois (1969)

[ 8] P HENRICI. Discrete varlable methods in ordinary differential equations, Wiley (1962).

[ 9] C.N.R S. Procedures Algol en analyse numerique. T 2, Chapitre 3, p 101, Editions du C.N.R S. (1970).

[10] P. SABLONNIERE Une méthode de resolution numérique de certaines equatıons intégrales de type Hammerstern. Colloque d'Analyse Numérique, Épinal (1972), Séminaire d'Analyse Numérique, Lille (1973). 